Corrigendum

August 2000

Volume 106 | Number 4

\title{
A novel signaling mechanism between gas and blood compartments of the lung
}

Wolfgang M. Kuebler, Kaushik Parthasarathi, Ping M. Wang, and Jahar Bhattacharya

J. Clin. Invest. 105:905-913 (2000).

During the preparation of this manuscript for publication, an error was introduced in Figure 7. The correct version, accompanied by the legend, appears below.
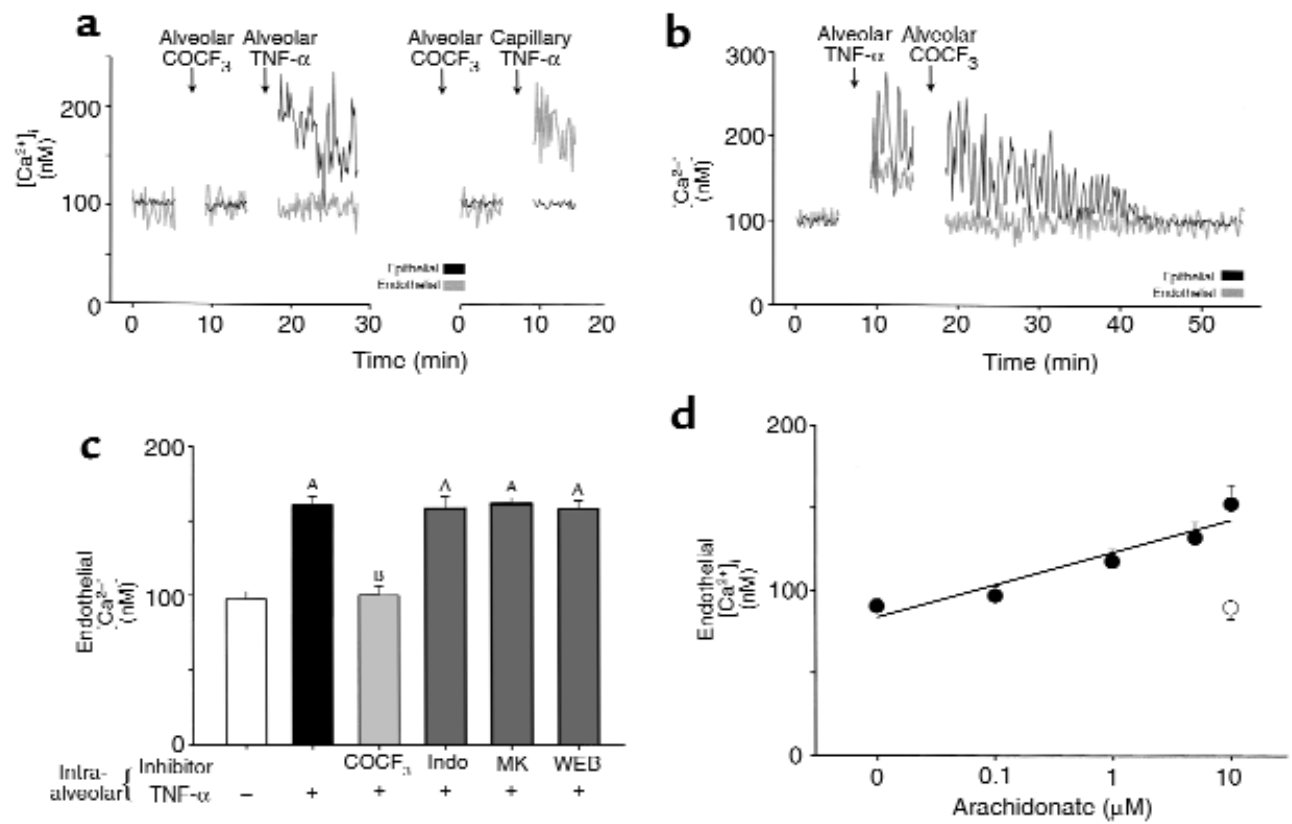

Figure 7

Effects of cPLA $A_{2}$ and its products. ( $\mathbf{a}$ and $\mathbf{b}$ ) Tracings from single experiments. Alveolar or capillary routes of TNF- $\alpha$ infusion are indicated. Replicated 4 times each. (c) Group data for indicated inhibitors given in 10-minute alveolar infusions before alveolar infusion of TNF- $\alpha ; n=4$ for each. ${ }^{A} P<0.05$ vs. baseline (open bar). ${ }^{\mathrm{B}} P<0.05$ vs. TNF- $\alpha$ (solid bar). $\mathrm{COCF}_{3}, \mathrm{AACOCF}_{3}$; indo, indomethacin; MK, MK886; WEB, WEB 2170BS. (d) Alveoli were permeabilized by 10-minute saponin infusions. Then arachidonate was infused into permeabilized (closed circles) and control (open circle) alveoli. Each point is the mean \pm SEM of 4 experiments. Line was drawn by linear regression $(P<0.001)$. 